\title{
A Statistical Local Binary Fitting Model for Blood Vessel Segmentation
}

\author{
Shifeng Zhao, Mingquan Zhou, Kang Wang \\ College of Information Science and Technology \\ Beijing Normal University \\ Beijing, China \\ zhaosf8109@gmail.com
}

\begin{abstract}
Network structure such as blood vessels in medical images are important features for computer-aided diagnosis and follow-up of many diseases. In this study, a new model-based segmentation method is proposed to detect blood vessels in medical images. The Local Binary Fitting(LBF) model with statistical distribution function is used for this purpose. The brain tissues and cerebral vessels in the image are modeled by Gaussian distribution and uniform distribution respectively. The region distribution combined with the LBF model is used in curve evolution. And the level set method is developed to implement the curve evolution to assure high efficiency of the cerebrovascular segmentation. Comparisons with the LBF method show that our model can achieve better results.
\end{abstract}

Keywords-segmentation; blood vessel; intensity inhomogeneity

\section{INTRODUCTION}

Blood vessel segmentation is important for clinical tasks such as diagnosis of vascular diseases, surgery planning and blood low simulation[1].And accurate automatic extraction of cerebrovascular system from images is a challenging segmentation problem due to small size objects of interest (blood vessels) and complex surrounding anatomical structures, e.g. fat, bones, or gray and white brain matter[2]

A lot of work has been devoted to vessels extraction in medical images. A complete survey is given by Lesage [3]. Holtzman-Gazit et al.[4] presented a segmentation method which combined minimal variance with the boundary alignment and the geodesic active surface for extracting thin structures in 3D medical images. Gooya et al. [5][6] proposed an energy edge detector for vessel segmentation using the structural tensor. Cohen and Deschamps [7]proposed the minimal path method to segment tubular structures. It was demonstrated that the front usually flows over the boundaries of longer and thinner objects when propagating. Therefore, they showed how the Fast Marching surface segmentation which can be specifically optimized for this target. Descoteaux et al. [8] proposed a boundary vessel segmentation by convolving an image with Gaussian filters at multiple scales to drives the shape evolution. And some recent relevant works along the same research line, like Law and Chung[9], where they present a vessel segmentation method based on weighted local variance and active contour model. Also, other interesting methods have been presented in Manniesing et al.[10], Nemitz et al. [11], Hernández Hoyos et al. [12], Orkisz et al. [13].
Recently,since the great success of curve evolution[14-17] and level-set method[18-24] in image segmentation, different approaches have been developed for extraction of blood vessel contours on medical images. For example, Tsai [15] used a set of deformable contours to define the boundaries between regions in an image and employ a gradient flow to evolve these contours. Li[24] put forward a region-based active contour model for image segmentation in a variational level set framework. Its good performance has attracted many attentions. However, the computational complexity is high for its convolution process, and its sensitive to the govering parameters and location of initial curve limit its applications. Deschamps[25] made use of Level-Sets methods to model the complex and varying surfaces of blood vessels.Farag[26] applied the level set to extract vascular tree in 3D volume. The level set balloons are manually initialized inside the $3 \mathrm{D}$ volume and then change with time to yield the blood vessels. And the segmenting results are much better than the 2D case.Mannidsing[27] put forward a method to use the background and vessel intensity distribution based on the intensity histogram and made use of the distribution to steer the level set to capture the vessel boundaries. And the better performance achieved made them come to the conclusion that the level set based vessel segmentation is a promising method. Also different hybird approaches attempt to combine the level set and other algorithm to get better results. For instance, a multiple scale method for segmenting blood vessels is derived in[28],by combing quadrature filters and local phase as the global information to drive the propagating fronts force.

Though many methods are proposed to extract the blood vessels using the level set method as much as possible, they did not take into account the problem of non-uniform property. Due to the disturbance of the noise and the volume effect in medical images, the intensity of different brain tissues overlap with each other. Also different imaging devices, and even the different imaging method of the same imaging device, the sensitivity and specificity of the different vascular are also different. So, it is reasonable to add the global information into the segmenting process. Acturally, in medial images, the intensity of various elements or components are also different, even the same tissue, they may take on different intensity and obey some certain distribution. Therefore, the development of a new vascular segmentation method was the prime motivation behind our work. In this study,we present an algorithm for segementing blood vessels based on a statistical model analysis and curve evolution method. 
The rest of this paper is organized as follows. In section 2, we briefly review the LBF model. In section 3, we describe our proposed model in detail. Section 4 is the implementation. In section 5, the comparations with LBF approach for real blood vessels are presented, which is followed by the conclusions given in Section 6 .

\section{LOCAL BINARY FITTING MODEL}

The Local Binary Fitting model proposed by Li et al[24] is composed of three terms, i.e., the fitting energy term, the penalty term and the length term. The energy functional can be described as:

$$
F^{L B F}\left(\phi, f_{1}, f_{2}\right)=E^{L B F}\left(\phi, f_{1}, f_{2}\right)+\mu P(\phi)+v L(\phi)
$$

where $\mu$ and $\nu$ are nonnegative constants.

The first term in (1) is defined as:

$$
\begin{aligned}
E^{L B F} & =\lambda_{1} \int\left[\int K(x-y)\left|I(y)-f_{1}(x)^{2}\right| d y\right] d x \\
& +\lambda_{2} \int\left[\int K(x-y)\left|I(y)-f_{2}(x)^{2}\right| d y\right] d x
\end{aligned}
$$

where $\lambda_{1}$ and $\lambda_{2}$ are positive constants, and $f_{1}(x)$ and $f_{2}(x)$ are two spatially varying fitting functions that fit image intensities near the point $X$.And $K$ is a Gaussian kernel with standard deviation.

The penalty term $P(\phi)$, which is used to ensure stable evolution of the level set function, is defined as:

$$
P(\phi)=\int_{\Omega} \frac{1}{2}(|\nabla \phi(x)|-1)^{2} d x
$$

And the length term $L(\phi)$ regularizing the zero level contour of $\phi$ is given by:

$$
L(\phi)=\int_{\Omega} \delta(\phi(x))|\nabla \phi(x)| d x
$$

The introduction of the local binary fitting energy with kernal functions enables the extraction of accurate local information. Utilizing the local intensity information which plays a key role in segmenting process, the LBF model has achieved promising results. However, due to the limitation of local property of the kernel function, the final result may introduce many local minimums. And the result is more dependent on the initialization of the contour. And crossing and occlusions in medical images are challenging problems that cannot be overcome by local search procedure. So we combine the global information with the LBF model to get better segmenting results.

\section{DESCRIPTION OF THE PROPOSED MOLDE}

The model proposed by $\mathrm{Li}$ et al.[24] is based on Mumford-Shan model, so it is still a piecewise constant. But actually, the intensity of various brain tissues is not necessarily consistent. They may be subject to different distribution. We analyzed different images and found that the histogram always has one peak near the low intensity region, which is not surprising since intensity inhomogeneity often occurs in medical images. The distribution characteristics are meaningful features which can facilitate segmentation. So in order to get better segmentation result, we extend the model in the following steps.

First, from the above analysis, we will assume that the image consists of two classes, vessel and non-vessel. Non-vessel class includes the low intensity region and is modeled by a Gaussian distribution. The vessel class includes arteries and is modeled by a uniform distribution. The distribution of the image can be expressed as a finite mixture of two classes: vessel and non-vessel:

$$
f(x)=P\left(x \mid \omega_{1}\right) P\left(\omega_{1}\right)+P\left(x \mid \omega_{2}\right) P\left(\omega_{2}\right)
$$

where, $f(x)$ is the total distribution of image pixels. $x$ is the intensity. $P\left(x \mid \omega_{1}\right)$ is the posterior distribution of non-vessel class. $P\left(x \mid \omega_{2}\right)$ is the posterior distribution of vessel class. $P\left(\omega_{1}\right)$ and $P\left(\omega_{2}\right)$ are the proportion of non-vessel and vessel in image respectively. A Gaussian distribution models non-vessel class as follows:

$$
P\left(x \mid \omega_{1}\right)=\frac{1}{\sqrt{2 \pi \sigma^{2}}} \exp \left(-\frac{(x-\mu)^{2}}{2 \sigma^{2}}\right)
$$

a uniform distribution models vessel class as follows:

$$
P\left(x \mid \omega_{2}\right)=\frac{1}{I}
$$

where $I$ is the maximum intensity in the image.

Second, we introduce the distribution into the LBF model, then we will get our new model, so the energy functional can be written as:

$$
\begin{gathered}
E(\phi)=F^{L B F}\left(\phi, f_{1}, f_{2}\right)+\left(\frac{1}{\sqrt{2 \pi \sigma^{2}}} \exp \left(-\frac{\left(f_{0}-\mu\right)^{2}}{2 \sigma^{2}}\right) P(\phi)\right. \\
\left.+\frac{1-P(\phi)}{I}\right) \sin c^{2}(\phi-k)
\end{gathered}
$$

where $P(\phi)$ is the proportion of non-vessel class in the image. $f_{0}$ is the given image, $\mu$ is the mean, $\sigma^{2}$ is the variance of the Gaussian distribution, $I$ is the maximum intensity in the image.

Once $\phi$ comes to a steady state, the evolving curve $C$ will separates the blood vessels form the background. From this purpose, we seek a law of evolution in the form:

$$
\phi_{t}=-\operatorname{grad} E(\phi) \text {. }
$$

The symbol 'grad' here denotes the gradient in the space $L^{2}(\Omega)$.Therefore, we get the following gradient flow equation:

$$
\begin{aligned}
\phi_{t}= & \mu\left(\nabla^{2} \phi-\operatorname{div}\left(\frac{\nabla \phi}{|\nabla \phi|}\right)\right)+v \delta(\phi) \operatorname{div}\left(\frac{\nabla \phi}{|\nabla \phi|}\right) \\
& -\delta(\phi)\left(\int K(x-y)\left|I(y)-f_{1}(x)^{2}\right| d y\right) \\
& -\delta(\phi)\left(\int K(x-y)\left|I(y)-f_{2}(x)^{2}\right| d y\right)
\end{aligned}
$$


$-\lambda\left[\left(\frac{1}{\sqrt{2 \pi \sigma^{2}}} \exp \left(-\frac{\left(f_{0}-\mu\right)^{2}}{2 \sigma^{2}}\right) P(\phi)+\frac{1-P(\phi)}{I}\right)\right]$

$*\left(\frac{\sin (2 \pi(\phi-k))}{\pi(\phi-k)^{2}}-\frac{2 \sin ^{2}(\pi(\phi-k))}{\pi^{2}(\phi-k)^{3}}\right)$

where

$\phi(0, x, y)=\phi_{0}(x, y)$ in $\Omega$.

and

$\frac{\partial \phi}{\partial \vec{n}}=0 \quad$ on $\quad \partial \Omega$.

\section{IMPLEMENTATION}

Since the parameters are critical in the evolution process, in our numerical experiments, the mean $\mu_{0}$ is chosen to be the intensity corresponding to the histogram maximum value. The variance $\sigma_{0}$ can be got from formula(5). The initial value for $p(\phi)$ is from the histogram of the image. we also normalize the given image $f$ as $f_{0}=\frac{f-f_{\min }}{f_{\max }-f_{\min }}$,where $f_{\max }$ and $f_{\min }$ are the maximum and the minimum values of the given image, respectively. When the distribution is Gaussian, the parameters of the distribution can be derived from the following:

$$
\begin{aligned}
& P^{(t)}\left(\omega_{k} \mid x_{i}\right)=\frac{P^{(t)}\left(x_{i} \mid \omega_{k}\right) P\left(\omega_{k}^{(t)}\right)}{\sum_{j=1}^{2} P^{(t)}\left(x_{i} \mid \omega_{j}\right) P\left(\omega_{j}^{(t)}\right)} \\
& P^{(t+1)}\left(\omega_{1}\right)=\frac{1}{N} \sum_{i=1}^{N} P^{(t)}\left(\omega_{1} \mid x_{i}\right) \\
& \mu_{k}^{(t+1)}=\frac{\sum_{i=1}^{N} P^{(t)}\left(\omega_{i} \mid x_{i}\right) x_{i}}{\sum_{i=1}^{N} P^{(t)}\left(\omega_{i} \mid x_{i}\right)} \\
& \left(\sigma^{(t+1)}\right)^{2}=\frac{\sum_{i=1}^{N} P^{(t)}\left(\omega_{1} \mid x_{i}\right)\left(x_{i}-\mu^{(t+1)}\right)^{2}}{\sum_{i=1}^{N} P^{(t)}\left(\omega_{1} \mid x_{i}\right)}
\end{aligned}
$$

\section{EXPERIMENTAL RESULTS}

Experiments have been carried out on real blood vessels. Figure 1 shows the segmentation results for three real blood vessel images with inhomogeneous intensity via use of the Local Binary Fitting model and the proposed method. It can be seen from the results that the proposed model can achieve better segmenting results.

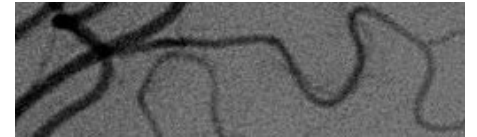

(a) original image

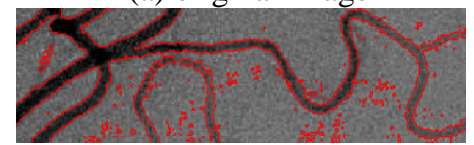

(b) LBF model

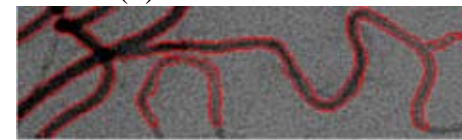

(c) the proposed model.

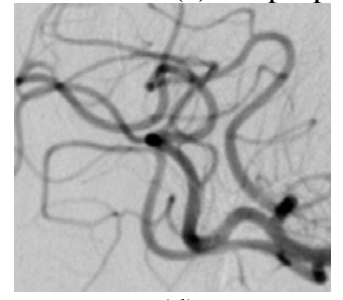

(d)

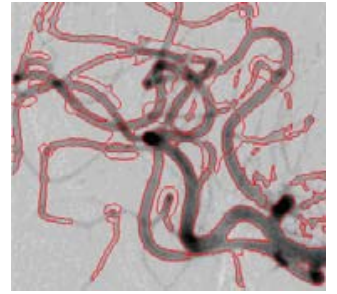

(f)

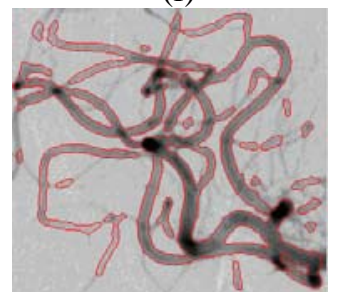

(i)

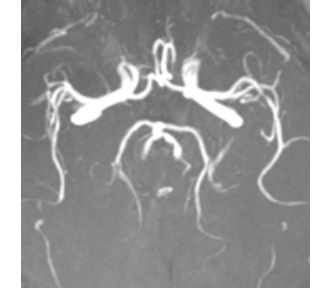

(e)

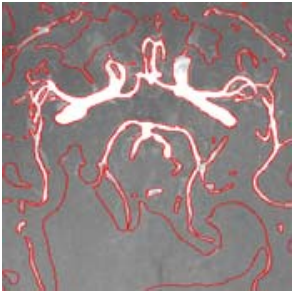

(g)

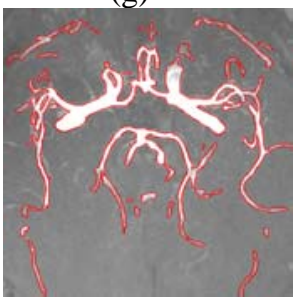

(j)
Figure 1 The comparisons of the LBF model and the proposed model on segmenting real blood image with the intensity inhomogeneity. (a)(d)(e)original images. (b)(f)(g) final segmentation result using LBF model. (c)(i)(j) final segmenting results using the proposed model.

Table 1 shows the evalution segmentation results of the propose method as well as the results yielded by the LBF method. In general, the results show that the new model based mthod perform better than the LBF approach.

TABLE I. THE PERFORMANCE COMPARATION OF THE TWO MODEL

\begin{tabular}{|l|c|c|c|c|c|c|}
\hline \multirow{2}{*}{} & \multicolumn{2}{|c|}{ size $=\mathbf{2 5 5}$ *77(a) } & \multicolumn{2}{c|}{ size $=\mathbf{3 1 9}$ *294(d) } & \multicolumn{2}{c|}{ size $=314 * 340(\mathbf{c})$} \\
\cline { 2 - 7 } & $\begin{array}{l}\text { iteration } \\
\text { number }\end{array}$ & $\begin{array}{l}\text { total } \\
\text { time }\end{array}$ & $\begin{array}{l}\text { iteration } \\
\text { number }\end{array}$ & $\begin{array}{l}\text { total } \\
\text { time }\end{array}$ & $\begin{array}{l}\text { iteration } \\
\text { number }\end{array}$ & $\begin{array}{l}\text { total } \\
\text { time }\end{array}$ \\
\hline $\begin{array}{l}\text { LBF } \\
\text { model }\end{array}$ & 20 & 16.7 & 50 & 52.2 & 100 & 88.9 \\
\hline $\begin{array}{l}\text { New } \\
\text { model }\end{array}$ & 18 & 14.8 & 40 & 32.6 & 80 & 66.4 \\
\hline
\end{tabular}

For the table above, we also come to that our proposed model has advantages in handling larger images. When dealing with large images, our method can get better results 
with less iteration number and less time.

\section{CONCLUSION}

The accurate segmentation of vessel network plays an important role in the analysis of vessel structure and topology. The presented method which combines the curve evolution with global statistical information can achieve a better segmentation result. By incorporting the local binary fitting energy and the global intensity distribution, most of the vessel network can be obtained. Compared with LBF method, the proposed method can improve efficiency and accuracy in the segmenting process. Future work will include reducing the computating complexity of the algorithm and tacking low constrast blood vessels.

\section{ACKNOWLEDGMENT}

The work was supported by the National Natural Science Fundation of China under Grant No.61170170 and No.61003134 and by Beijing Municipal Natural Science Foundation under Grant No.4081002.

\section{REFERENCES}

[1] Delphine Nain, Anthony J. Yezzi, Greg Turk. Vessel Segmentation Using A Shape Driven Flow. Medical Image Computing and Computer-Assisted Intervention - MICCAI , pp. 51-59, 2004.

[2] Ayman El-Baz, Georgy Gimel'farb, Ahmed Elnakib, Robert Falk, and Mohamed Abou El-Ghar.Fast, Accurate Unsupervised Segmentation of 3D Magnetic Resonance Angiography. Atherosclerosis Disease Management, 2011, pp 411-432.

[3] Lesage, D., Angelini, E. D., Bloch, I., and Funka-Lea, G. A review of 3D vessel lumen segmentation techniques: models, features and extraction schemes. Medical Image Analysis, 13(6),pp. 819-845,2009.

[4] Holtzman-Gazit, M., Kimmel, R., Peled, N., and Goldsher, D. Segmentation of thin structures in volumetric medical images. IEEE Transactions on Image Processing, 15, pp.354-363, 2006.

[5] Gooya, A., Liao, H., Matsumiya, K., Masamune, K., Masutani, Y., and Dohi, T. A variational method for geometric regularization of vascular segmentation in medical images. IEEE Transactions on Image Processing, 17(8),pp. 1295-1312,2008.

[6] Gooya, A., Dohi, T., Sakuma, I, and Liao, H. R-PLUS: a Riemannian anisotropic edge detection scheme for vascular segmentation. In MICCAI'08: Proceedings of the 11th international conference on medical image computing and computer-assisted intervention-Part I (pp. 262-269). Berlin: Springer.

[7] Cohen, L. D., and Deschamps, T. Segmentation of 3D tubular objects with adaptive front propagation and minimal tree extraction for 3D medical imaging. Computer Methods in Biomechanics and Biomedical Engineering, 10(4), pp.289-305, 2007.

[8] M. Descoteaux, L. Collins and K. Siddiqi. A Multi-Scale Geometric Flow for Segmenting Vasculature in MRI : Theory and Validation. Medical Image Analysis,12(4), pp.497-513. August 2008.

[9] Law, M. W. K., and Chung, A. C. S. Weighted local variancebased edge detection and its application to vascular segmentation in magnetic resonance angiography. IEEE Transactions on Medical Imaging, 26(9), pp.1224-1241, 2007.

[10] Manniesing, R., Viergever, M. A., and Niessen, W. J. (2007). Vessel axis tracking using topology constrained surface evolution. IEEE Transactions on Medical Imaging, 26(3), pp.309-316, 2007.

[11] Nemitz, O., Rumpf, M., Tasdizen, T., and Whitaker, R. Anisotropic curvature motion for structure enhancing smoothing of 3D MR angiography data. Journal of Mathematical Imaging and Vision, 27(3), pp. 217-229. 2007.
[12] Hernández Hoyos, M., Serfaty, J. M., Maghiar, A., Mansard, C., Orkisz, M., Magnin, I. E., \& Douek, P. Evaluation of semiautomatic arterial stenosis quantification. International Journal of Computer Assisted Radiology, 1(3), pp.167-175, 2006.

[13] Orkisz, M., Flórez Valencia, L., and Hernández Hoyos,M. Models, algorithms and applications in vascular image segmentation. Machine Graphics and Vision, 17(1), pp.5-33, 2008.

[14] Jayant Shah, A Common Framework for Curve Evolution,Segmentation and Anisotropic Diffusion. IEEE Conference on Computer Vision and Pattern Recognitioni, 1996,136-142.

[15] Andy Tsai and Anthony J. Yezzi Jr. and Alan S. Willsky .Curve evolution implementation of the Mumford-Shah functional for image segmentation, denoising, interpolation, and magnification. IEEE Transactions on Image Processing. 2001,1169-1186.

[16] Yongsheng J, Pan, Douglas Birdwell, Seddik M. Djouadi, Image Segmentation Using Curve Evolution and Anisotropic Diffusion: An Integrated Approach, Seventh IEEE International Symposium on Multimedia (ISM'05), 2005 ,387-394.

[17] Andy Tasi, Anthony Yezzi, Jr, Curve evolution implementation of the Mumford-Shah Functional for image segmentation, denoising, interpolation and magnification. IEEE transactions on image processing vol.10.No.8.August,2001,1169-1186.

[18] L. M. Lorigo, O. D. Faugeras, W. E. L. Grimson, R. Keriven, R. Kikinis, A. Nabavi, C.-F. Westin,CURVES: Curve Evolution for Vessel Segmentation,Medical Image Analysis, 5, 2001,195-206.

[19] Tony F. Chan, Luminita A. Vese. Active contours without edges. IEEE Transactions on Image Processing, 10(2) ,2001,: 266-277.

[20] Luminita A. Vese, Tony F. Chan. A Multiphase level set framework for image segmentation using the Mumford and Shah model. International Journal of Computer Vision 50(3), 2002,271-293.

[21] Tai X Ch,Chan TF.A survey on multiple level set methods with applications for identifying piecewise constant functions[J].International Journal of Numerical Analysis and Modeling,2004,1(1):25-47.

[22] Chung, G., Vese, L.A.: Energy minimization based segmentation and denoising using multilayer level set approach. LNCS, 3757, 439-455 (2005).

[23] Lie, Johan.; Lysaker, Marius.; Tai, Xue Cheng. A variant of the level set method and applications to image segmentation. Mathematics of Computation. 75, 2006:1155-1174.

[24] C.M. Li, C. Kao, J. Gore, Z. Ding, Implicit Active Contours Driven by Local Binary Fitting Energy, Proceedings of IEEE Conference on Computer Vision and Pattern,2007.

[25] Deschamps, T., Schwartz, P., Trebotich, D., Colella, P., Saloner, D., Malladi, R.: Vessel segmentation and blood flow simulation using Level-Sets and Embedded Boundary methods. ;In CARS(2004)75-80.

[26] Aly A. Farag,Hossam Hassan, Robert Falk and Stephen G.Hushek.3D Volume Segmentation of Mra Data Sets Using Level Sets. Academic radiology, 11(2004) ,419-435.

[27] R.Manniesing,B.K. Velthuis, M.S. van Leeuwen,I.C. van der Schaaf,P.J.van Laar, W.J. Niessen. Level set based cerebral vascularture segmentation and diameter quantification in CT angiograph. Medical Image Analysis.10(2006),200-214.

[28] Gunnar Läthén, Jimmy Jonasson and Magnus Borga. Phase Based Level Set Segmentation of Blood Vessels. International Conference on Pattern Recognition,2008,1-4. 